\title{
On the arithmetization of algebraic geometry
}

\author{
Silke Slembek \\ Institut de Recherche Mathématique Avancée \\ Université Louis Pasteur et CNRS \\ 7 , rue René Descartes \\ F-67084 Strasbourg Cedex \\ France
}

The arithmetization of algebraic geometry was one of the efforts to give new foundations to the field by rewriting (parts of) it using the language of modern algebra and arithmetical ideal theory. It was initiated and named by Oscar Zariski in the late 1930s.

This approximate description of arithmetization is of course too general to see clearly the characteristic patterns concerning its motivations, structure, and objectives.

The main goal of this chapter is to show the features of this foundational process and to suggest an interpretation. I will argue that one of its main goals was to legitimize algebraic geometry as it was practiced by Italian algebraic geometers since the end of the XIXth century. Consequently it had strong conservative tendencies. At the same time arithmetization was an innovative process that entailed a complete reshaping of the discipline.

\section{Different rewritings of algebraic geometry}

By 1900 different approaches to algebraic geometry had been established. The one that was - as well by its authors as by others - called the Italian approach was shaped among others by Guido Castelnuovo and Federigo Enriques. They forged a new approach to algebraic geometry in the late XIXth century, inspired by Brill and Noether [1874], Veronese [1882], and Segre [1894].

In their 1874 paper "Ueber die algebraischen Functionen und ihre Anwendung in der Geometrie" Alexander von Brill and Max Noether promoted the use of algebraic methods in the theory of abelian functions introduced by Riemann in 1851 and 1857 and applied to geometry by Clebsch in 1864 . They formulated the theory of algebraic functions in the language of linear series and based their rewriting on the $A f+B g$ theorem. Thus transcendental considerations (in particular the Dirichlet principle) were avoided and the theory was made a branch of algebra [Brill and Noether 1874, p. 269].

Castelnuovo and Enriques generalized the theory of algebraic curves developed by Brill and Noether to algebraic surfaces in their 1897 paper "Sur quelques 
récents résultats dans la théorie des surfaces algébriques." Contrary to Brill and Noether, they played down algebra and tried to give a purely geometric treatment. Their approach was deeply influenced by the use that Giuseppe Veronese had made of higher dimensional geometry and by works of Corrado Segre. Veronese showed in his [1882] that it was often useful to look at geometric objects as projections of some objects in a higher dimensional space. For instance, he showed that a rational plane curve of order $m$ is the projection of a "normal" curve of order $m$ belonging to the $m$-dimensional projective space. In particular, certain singular curves could be regarded as non-singular curves in a suitable projective space.

Segre had taken up the idea of Veronese and proposed to look for families of curves that yield an embedding of a surface in some suitable projective space. Although Segre was at the same time much influenced and inspired by Brill and Noether, he promoted a different approach to algebraic geometry. In his treatise Introduzione alle geometria sopra un ente algebrico semplicemente infinito in 1894 he abandoned the fundamental theorem of Brill and Noether. Instead, he intended to build the theory of linear series of points solely on geometric considerations. Moreover, he emphasized the birational point of view when he defined in 1894: "We can now easily define the object of the geometry on a variety $M_{k}$. It is the geometry that studies those properties of the $M_{k}$ that are invariant under birational transformations of the variety." [Segre 1894 or 1954 , p. 210]. As far as curves were concerned, his program was to translate the properties of a curve that are invariant under birational transformation into projective properties of an appropriate model of the curve. Castelnuovo and Enriques carried out Segre's program for algebraic surfaces.

Although other approaches to the study of algebraic surfaces were developed parallel to the development in Italy, algebraic geometry was largely identified with the geometric approach promoted by Italian mathematicians. In the 1920s one of the most productive research centers still was Rome. Many mathematicians went to there to work with the masters and to learn algebraic geometry on the spot.

Oscar Zariski (1899-1986), on the contrary, enrolled at the university of Rome in 1921, he received his mathematical training and culture from Castelnuovo who was his thesis advisor, from Enriques who encouraged his first publications, and from Severi. So that he became part of the Italian school and was regarded as one of the most promising figures of the new generation of mathematicians trained in Italy.

\section{Criticism}

It was also during the 1920 s that criticism arose concerning the methods of Italian algebraic geometry. When mathematicians of the Italian school were confronted with the critique, several tried to defend the school. Severi for example defended the methods and standards of the Italian school against the demands of modern algebra and topology by making a subtle difference between the rigor of the working mathematician and the formal rigor of proofs [Severi 
1949]. The 20 years younger Zariski joined the critiques and invented a new approach to algebraic geometry in order to show that "our fathers were right" [Zariski 1950, p. 78 or 1978 , p. 364].

Zariski encountered the critique on different occasions and in different ways. The first hint to the need of new methods might have come from inside the Italian school itself : from Castelnuovo who had looked for new methods, and turned to analysis situs after 1910. He encouraged Zariski to learn and use the new tools.

Castelnuovo, Enriques, and Severi sent Zariski to the United States to work with Salomon Lefschetz in 1927. For about 10 years his research concentrated on topological properties of algebraic curves (fundamental group). One can assume that he knew Lefschetz' point of view about Italian algebraic geometry. Namely that it was "magnificent" but far too specialized, meaning that the methods were taylor-made for specific problems and needed a huge amount of practice. But it was certainly not Lefschetz who pushed Zariski towards modern algebra. According to him, it should be possible to recover the results of the Italians with more or less elementary methods of analysis. ${ }^{1}$

Finally, he came to formulate his own critique when he was writing a monograph on the theory of Algebraic surfaces, the core topic of Italian algebraic geometry. It was published 1935 in the series Ergebnisse der Mathematik und ihrer Grenzgebiete. The book intended to give a systematic exposition of the basics of the theory. To give this exposition, Zariski had to peruse large parts of the existing literature on algebraic surfaces. When reviewing the contributions of Italian geometers, Zariski came across gaps, errors, and weaknesses. Many were tacitly corrected, others were openly criticized. Still, as BrigagliaCiliberto rightly observed and as a closer analysis confirms, the lack of rigor is sometimes criticized, but rigor is not the main issue here [Brigaglia-Ciliberto 1995, p. 121]. One problem seems to retain special interest: the resolution of singularities of algebraic surfaces. The question if any given algebraic surface can be transformed with finitely many birational transformations into a surface without singularities was intensely studied by Italian geometers in the 1890s (See [Gario 1988, 1989]). Between 1892 and 1924 several proofs have been given to that theorem. Zariski reviews each of them because "in the theory of singularities the details of the proofs acquire a special importance and make all the difference between theorems which are rigorously proved and those which are only rendered highly plausible" [Zariski 1935, p. 18]. The conclusion of the perusal is clear: several proofs have been proposed by Italian geometers and none of them stands critical examination. The theorem is thus rendered "highly plausible" but not "rigorously proved".

There were so many points criticized throughout the monograph that Lefschetz pointed out in his review of the book (for the Bulletin of the AMS) that the reader should not get the "unjust impression that Zariski occupied himself chiefly with a critique of things long since done. As a matter of fact this critique takes up only half of the monograph, the rest being given over to modern

\footnotetext{
${ }^{1}$ Letter to Weyl, ETHZ. I thank David Rowe for pointing this letter out to me.
} 
questions."

Lefschetz' remark reflects the image of Italian algebraic geometry one could get from reading Algebraic Surfaces. Indeed, from Zariski's choice of the literature the study of algebraic surfaces seems to have been a vital topic between 1890 and 1910, and during that period th was dominated by Italian mathematicians. After World War I the scene was profoundly altered: the Italian school seems to contribute little, research activities moved on to other european countries and to America instead. So the parts of the theory that were criticized were the ones "long since done". But those are the results and proofs by Italian geometers. Thus the image of Italian algebraic geometry being old-fashioned and not quite meeting contemporary standards for mathematical writing was fostered.

The other part of the quote from Lefschetz gives a hint to the alternatives to the Italian algebro-geometric approach that were perceived. Zariski announced in the preface of Algebraic Surfaces that the exposition should emphasize "the interrelations between the various aspects of the theory: algebro-geometric, topological, transcendental" [Zariski 1935, Preface]. In fact, six chapters out of eight deal with the traditional, Italian algebro-geometric approach, one with the transcendental one and one with the topological approach. The standpoint of modern algebra had not yet permeated algebraic geometry to the point of being included among the modern approaches. But among the promising modern questions were those concerning topological properties of algebraic surfaces. This is exactly the field that Zariski (influenced by Lefschetz) was working in. Nothing in the book indicates that Zariski was about to leave this field and wanted to rewrite algebraic geometry arithmetically. He concentrated his research on topological questions (fundamental group) and topological methods that seemed modern, "clean and new" [Mumford 1986, p. 891].

When he decided to launch another rewriting of algebraic geometry (besides the one van der Waerden started in the late 1920s and that Zariski learned about in the early 1930s) he turned to modern algebra and arithmetic ideal theory. Again, he had to get acquainted with the tools. When he had left Italy, he did not know anything about modern algebra. "If somebody had asked me, 'What's a ring?' I couldn't have defined it."' Zariski new van der Waerden's book Moderne Algebra soon after it was published in $1930 / 31$. Shortly after writing Algebraic Surfaces Zariski was invited to Princeton, where he heard lectures by Emmy Noether. But it was only in Krull's Idealtheorie (1935) that he found concepts that he could interpret geometrically and that offered a basis for the rewriting of algebraic geometry. At the end of the 1930s the arithmetization finally began.

Within a little more than 10 years, the new methods replaced the old ones. By the middle of the XXth century Italian algebraic geometry has lost almost all its impact while Oscar Zariski who became professor at Harvard in 1947 became the "point of reference for the new generation of algebraic geometers". The new

\footnotetext{
${ }^{2}$ Quoted in: [Parikh 1991, p. 68]. This was not typical for the training in Italy, Dieudonné made a similar remark: "I had graduated from the École Normale, and I did not know what an ideal was, and I barely knew what a group was." Quoted in: [Corry 1996, p. 309].
} 
american school was influential through Zariskis students such as David Mumford, Heisuke Hironaka, Shreeram Abhyankar, Joseph Lipman and others.

\section{The starting point of arithmetization}

Arithmetization was a reaction to several criticisms about Italian algebraic geometry - criticism coming to a large extent from Zariski himself. Where did it start and why? The answer to these questions will elucidate the motivation of the process and give some insight to its initial design.

"After spending a couple of years just studying modern algebra, I had to begin somewhere" Zariski explained [Zariski 1972, xi]. But the starting point was not as arbitrary as "somewhere" might suggest. In fact, the starting point was the problem of the resolution of singularities, particularly important to Italian geometers and identified by Zariski as particularly insufficiently proved.

In order to understand why this proof became the touchstone for the arithmetization, it will be helpful to give some background about the theorem.

The analogous theorem for plane algebraic curves was first stated by Max Noether in 1871: every plane algebraic curve is birationally equivalent to a plane algebraic curve having only "ordinary" singularities (double points with distinct tangents and cusps). The transformation can be carried out with a finite number of suitable quadratic transformations of the projective plane. Suitable means here a quadratic transformation that has a fundamental point in a singularity of the given curve. The series of quadratic transformations yields a decomposition of the singular point.

Twenty years later the question was taken up and generalized to algebraic surfaces by Italian mathematicians. Segre gave a proof of the assertion that the process of decomposition of an isolated singularity on an algebraic surface was always finite, except in the case when the singular point lies on a multiple component of the surface. To Segre, this seemed "intuitively" clear. He was vividly criticized by del Pezzo for its use of an analytic result of Kobb (See [Gario 1988, 1989]). Segre's student Beppo Levi gave a geometric proof of this result in 1897. In the same year he published a proof of the resolution problem for the singular locus of an algebraic surface. Levi took up (like every successful proof of the resolution theorem) Noether's idea of using certain Cremona transformations having a fundamental point at a singularity $P$ of the surface $F$. What happens to the surface under such a transformation? Levi analyzed the situation as follows: to the singular $s$-fold point $P$ correspond on the transformed surface $F^{\prime}$ either finitely many points with multiplicity $s$ or infinitely many such points all lying on a curve. In the second case appears a curve included in the singular locus of the transformed surface. Depending on which case occurs Levi applied different types of Cremona transformations. In the first case he had to resolve isolated points with multiplicity $s$. This can give rise to a finite series of infinitely near points - in which case he's done. But during the resolution of an isolated point might also apprear a curve of multiplicity $s$ which brings him to the second case above again. As seen in retrospect, Levi's argument that an $s$-fold curve can be resolved is not understandable. Moreover new singularities are introduced at 
each transformation: so called "accidental singularities". Levi obviously thought that they were easy to get rid of - but again, his argument is not clear at all.

Today, the status of the proof is undecidable. But Levi's contemporaries did not doubt the correctness of the argument and accepted the proof as valid and rigorous. Other proofs were given by Severi (1914), Albanese (1921), Chisini (1924) with the aim of increasing its simplicity or elegance.

A little more than ten years after Chisini's proof two more proofs were given by Robert J. Walker and by Zariski. Their aim was radically different: they explicitly wanted to replace the proof given by Levi because it did not seem sufficient any longer. In 1935 Lefschetz' student Walker carried out part of Lefschetz program that I mentioned earlier (namely to build a theory parallel to the geometric one within "the grasp of an average analyst") in his $\mathrm{Ph}$. D. thesis and gave an analytic proof of the resolution theorem. Walker explicitly motivated his work with the desire to have a "completely rigorous proof for a theorem which is of such fundamental importance in the theory of surfaces". Moreover, the proof "should make use of as few as possible of the peculiar properties of algebraic surfaces" - which seems to echo Lefschetz' call for less specific methods. Finally the methods should extend to higher dimensional varieties [Walker 1935, p. 336].

While Walker did not go any further with any kind of criticism, Oscar Zariski did. As I mentioned earlier, in his monograph Algebraic surfaces (1935) he reviewed all geometric proofs given so far and rejected them all. He thus showed that there was no valid proof of the theorem of resolution of singularities using geometric methods. The proof by Walker on the contrary "stands the most critical examination and settles the validity of the theorem beyond any doubt" as Zariski wrote in a footnote added in print [Zariski 1935, p. 23]. So finally, the resolution theorem was more than "rendered highly plausible" — it was rigorously proved. But it was still not proved within the geometric framework. This is a crucial point. What triggered off arithmetization was not the need for a proof in order to give a rigorous foundation for some result. Such a proof already existed.

We can be more precise now about the motivation of the arithmetization process. It was designed to give a new legitimation of algebraic geometry. To legitimize algebraic geometry means - among other things - to show that its central results can be proved by using its own methods and not analytical ones.

The arithmetization of algebraic geometry was first stated as a research aim in an article published in 1938 "On the polynomial ideals defined by infinitely near base points," In this article Zariski isolated the parts of Italian algebraic geometry that he intended to build on. Furthermore he gave a description of the structure he was planning to give to the new theory.

He started from "the foundation of a geometric theory of singularities of algebraic surfaces," namely the concept of infinitely near points [Zariski 1935, p. 13].

The concept was introduced by Riemann and given a precise meaning by $\mathrm{M}$. Noether. This was done in 1871 and 1875 when M. Noether wanted to replace the analytic methods used throughout the XIXth century in the analysis of sin- 
gularities of algebraic curves, namely Puiseux series expansions, by algebraic processes. The main result of his articles is the theorem we mentioned earlier. Infinitely near points occur in the reduction process. Noether used a quadratic transformation of the projective plane that we may write (in homogeneous coordinates)

$$
\left(x_{0}: x_{1}: x_{2}\right) \mapsto\left(x_{1} x_{2}: x_{0} x_{2}: x_{0} x_{1}\right)
$$

This transformation has the three fundamental points $(1,0,0),(0,1,0)$ and $(0,0,1)$ that are mapped to the lines $L_{i}=\left\{x_{i}=0\right\}$. Conversly, the three fundamental lines $L_{0}=\left\{x_{0}=0\right\}, L_{1}=\left\{x_{1}=0\right\}, L_{2}=\left\{x_{2}=0\right\}$ are contracted to the points $(1,0,0),(0,1,0)$, and $(0,0,1)$ respectively. Let $C$ be a plane algebraic curve having an $s$-fold point at the fundamental point $O=(1,0,0)$ of the transformation. Under the transformation, the curve $C$ is transformed into a plane curve $\tilde{C}$. The point $(1,0,0)$ maps to the line $L_{0}=\left\{x_{0}=0\right\}$. To the singular point $O$ of $C$ correspond on $\tilde{C}$ the intersection points $O_{1}, O_{2}, \ldots$ of $\tilde{C}$ with $L_{0}$, which means that the $s$-fold point is decomposed into a finite number of points with smaller multiplicity.

The points $O_{1}, O_{2}, \ldots$ which have multiplicity smaller than the multiplicity of $O$ constitute the first neighborhood of $O$ and are infinitely near points of the first kind of $O$. They need not all be simple. If $O_{1}$ is not simple, the resolution process is repeated with a quadratic transformation having a fundamental point in $O_{1}$ giving rise to the second neighborhood of $O$ formed by the points $O_{1_{1}}, O_{1_{2}}, \ldots$ Noether's result states that a finite series of quadratic transformations affords a curve that is birationally equivalent to $C$ and has only ordinary singularities. At each step, the points $O_{i_{1}, i_{2}, \ldots i_{j}}$ are called infinitely near points of kind $j$ forming the $j$-th neighborhood of $O$. If the process is reversed, the singular point can be seen as composed of a finite series of infinitely near points.

In the 1897, the concept of infinitely near points was generalized by Corrado Segre to algebraic surfaces. In an article on the decomposition (scomposizione) of the singular points of algebraic surfaces he tied up with Noether's notion and the perception of a singular point as composed of a finite number of infinitely near points. He gave a rigorous definition of infinitely near points of an algebraic surface (See [Gario 1988, 1989]).

The other tool Zariski took from Italian algebraic geometry was the notion of a linear system. A linear system of curves on a surface $F$ is the family of curves cut out on $F$ by a system of hypersurfaces of the surrounding space of the form

$$
\left\{\lambda_{1} F_{1}+\lambda_{2} F_{2}+\ldots+\lambda_{r} F_{r}=0 \mid \lambda_{1}, \ldots, \lambda_{r} \text { complex numbers }\right\}
$$

where $F_{1}, \ldots, F_{r}$ are homogeneous polynomials of the same degree. The importance of linear systems lies (and lied) in the fact that they yield an embedding of the surface by mapping a point $x_{0}, \ldots, x_{n}$ of $F$ to the point $\left(F_{1}\left(x_{0}, \ldots, x_{n}\right), \ldots,-\right.$ $F_{r}\left(x_{0}, \ldots, x_{n}\right)$. The linear system of all conic sections for example that Veronese used in [1882] yields an embedding

$$
\left(x_{0}: x_{1}: x_{2}\right) \mapsto\left(x_{0}^{2}: x_{1}^{2}: x_{2}^{2}: x_{1} x_{2}: x_{0} x_{2}: x_{0} x_{1}\right)
$$


of the projective plane in the projective space of dimension 5 .

These are the geometric concepts that arithmetization was to rely on.

The link between the two concepts was thought of in the following way. A linear system could be defined by base conditions: one can require that the curves of the system pass through a point, a set of points or even through infinitely near points. In the first case the arithmetic translation is that the equations of the curves belong to the ideal of the point. In the second case, they should all belong to the intersection of the ideals of the points. In the third case one must replace the ideals of the points by co-called $v$-ideals. Zariski observed that the ideals that are of interest in this context are complete ideals; that is, ideals that can be written as the intersection of $v$-ideals. The terminology is chosen expressedly to reflect the analogy with the geometric notion of complete linear systems in the sense of linear systems that are uniquely defined by base conditions.

At this point the process of arithmetization was described as a reformulation of the existing theory. As Zariski put it "It is the main purpose of the present investigation to develop an arithmetic theory parallel to the geometric theory" [Zariski 1938, p. 151 or 1972 , p. 14]. Zariski insisted on the terminological parallel, see [Zariski 1939a, p. 151, 170, 193, 197 or 1972, p. 14, 33, 56, 60]. But constructing the arithmetic theory "along the lines" of the geometric theory does not only mean using the same words (although this is an interesting feature). The parallelism manifests itself in other ways. For example, the structure is taken over; that means the decision about what the basic notions are and the argumentation in the presentation of the theory. Furthermore, the limits of the geometric theory are accepted. This means that for example properties of the simple or complete $v$-ideals only reflect geometric properties. Thus, there is no surprise in arithmetization at this points - at least not for somebody trained in the Italian tradition.

This parallelism was desirable and possible only because the geometric theory was accepted as a reliable, rigorous, reasonable basis for the study of algebraic curves and surfaces. This means that rigor was not an issue here. So the new theory need not be a complete restart, a tabula rasa but it could rely on some existing foundations.

\section{Leaving the beaten track}

But Zariski could not realize the resolution solely by following the traditional lines. He also had to go beyond the existing theory and invent new concepts and a new operation. This was done in the articles "Some results in the arithmetic theory of algebraic varieties" and "The resolution of the singularities of an algebraic surface" that appeared in the American Journal of Mathematics (vol. 61, 1939) and in the Annals of Mathematics (vol 40. 1939) respectively. The "arithmetic theory of algebraic varieties" introduces the concept of arithmetically normal variety and the operation of "deriving" such varieties from an arbitrary variety. These tools are used to give an arithmetic proof of the resolution of the singularities of an algebraic surface. Zariski still tied the arithmetic 
theory up with the geometric theory that was produced in Italy [Zariski 1939a, p. 251 and p. 258 et sqq. or 1972 , p. 75 and p. 82 et sqq.]. But the parallel between the theories is now expressly given up - as well in the structure as in the concepts (See [Zariski 1939a, p. 288 or 1972, p. 112]).

The concept of an arithmetically normal variety was inspired by the existing notion of normal varieties. Since the 1880s normal varieties were defined as varieties which are not the projection of a variety of the same order belonging to a higher dimensional projective space. Zariski invented an arithmetical concept of normality. To begin with, the very concept of "variety" adopted by Zariski was different from the one mainly used before. An affine algebraic variety was thought of as the locus of zeros of homogeneous polynomials. Zariski adopted the point of view van der Waerden promoted in his works. From this viewpoint an irreducible algebraic variety $V$ is given by its generic (or general) point $\left(\xi_{1}, \ldots, \xi_{n}\right)$. The notion of "generic point" was inspired by Emmy Noether's definition of the "generic zero" (allgemeine Nullstelle) of a prime ideal. If $k$ is a field, $\Sigma=k\left(\xi_{1}, \ldots, \xi_{n}\right)$ a field extension, the polynomials $f\left(x_{1}, \ldots, x_{n}\right)$ that vanish at $\left(\xi_{1}, \ldots, \xi_{n}\right)$ form a prime ideal $\mathfrak{p} \subset k\left[x_{1}, \ldots, x_{n}\right]$. On the other hand, if $\mathfrak{p}^{\prime}$ is any prime ideal in $k\left[x_{1}, \ldots, x_{n}\right]$ then there exists a field extension $\Sigma=$ $k\left(\xi_{1}, \ldots, \xi_{n}\right)$ such that $\mathfrak{p}^{\prime}$ consists of all polynomials that vanish in $\xi_{1}, \ldots, \xi_{n}$. This field is unique up to isomorphism. Noether called $\left(\xi_{1}, \ldots, \xi_{n}\right)$ a "generic zero" of the ideal $\mathfrak{p}$. Van der Waerden interpreted this as the generic point of the variety $V$ whose ideal is $\mathfrak{p}$.

Zariski defined an algebraic variety to be arithmetically normal if the ring $\mathfrak{o}=k\left[\xi_{1}, \ldots, \xi_{n}\right]=k\left[x_{1}, \ldots, x_{n}\right] / \mathfrak{p}$ (the ring of homogenous coordinates as it is called today) is integrally closed in its quotient field $k\left(\xi_{0}, \ldots, \xi_{n}\right)$. It took Zariski a lot of technical work to show that the projective case is analogous.

What is the point of this definition? The manifold of singular points of an arithmetically normal variety of dimension $r$ in the projective space is of dimension at most $r-2$. So the only singularities that an arithmetically normal surface can have are located at isolated singular points. In particular, there are no curves included in the singular locus of the ring.

Thus, it is clear that those surfaces should play an essential role in the resolution process. If one had only to deal with arithmetically normal surfaces the problem would be considerably simplified. So, what is needed is an operation that transforms a given algebraic surface into an arithmetically normal one. This operation was introduced in the framework of the arithmetic theory of algebraic varieties to "establish the existence of (arithmetically) normal varieties in any given class of birationally equivalent varieties." [Zariski 1939a, p. 290 or 1972, p. 114]. Zariski called it "derivation of normal varieties" - this is what we call the normalization of varieties, the operation of taking the integral closure of the coordinate ring. The method is the following: The integral closure $\overline{\mathfrak{a}^{*}}$ of the homogeneous quotient ring $\mathfrak{o}^{*}=K\left[\xi_{0}^{*}, \xi_{1}^{*}, \ldots, \xi_{n}^{*}\right]$ is a finitely generated $\mathfrak{o}^{*}-$ modul, so it can be written as $\overline{\mathfrak{o}^{*}}=K\left[\zeta_{1}^{*}, \ldots, \zeta_{h}^{*}\right]$ where $\zeta_{1}^{*}, \ldots, \zeta_{h}^{*}$ have positive degree. But the elements $\zeta_{1}^{*}, \ldots, \zeta_{h}^{*}$ are not necessarily of the same degree, so Zariski could not see them as the homogeneous coordinates of some embedded projective variety. Zariski then shows the existence of a "character of homo- 
geneity", that is an integer $\delta$ such that the graded ring $\overline{\mathfrak{o}^{*}}(\delta)=\oplus_{\rho=1}^{\infty}\left(\overline{\mathfrak{o}^{*}}\right)_{\delta \rho}$ is gerated in degree one, that is by elements in $\overline{\mathfrak{o}}^{*}(\delta)_{1}=\left(\overline{\mathfrak{o}^{*}}\right)_{\delta}$. This operation has no analog in the geometric theory: Zariski takes the concept of integral closure of a ring in its quotient ring from algebra and arithmetic ideal theory, gives it a geometric interpretation and introduces in the theory of algebraic surfaces to solve the problem of reduction of singularities.

\section{The arithmetic proof}

With the concept of an arithmetically normal surface and the operation of deriving such surfaces from any algebraic surface at his disposal, Zariski could give a new proof of the reduction theorem. We briefly indicate the structure and the crucial arguments in this proof. ${ }^{3}$

From an arbitrary algebraic surface $\tilde{F}$, Zariski derives an arithmetically normal surface $F$. The surface $F$ has only isolated singularities. Compared to any other previous geometric proof, the operation of deriving arithmetically normal surface therefore makes a decisive difference.

Take one of the finitely many singular points of $F$, say $P$, and apply a quadratic transformation that has a fundamental point at $P$. To construct this transformation Zariski considered all quadratic forms that vanish in $P$. The elements of a base of these forms (over $k$ ) can be specialized at the generic point of $F$ and then be regarded as the homogeneous coordinates of the general point of an algebraic surface $F^{\prime}$. This transformation has only one fundamental point, namely $P$. To $P$ corresponds a fundamental curve $\Gamma$ on $F^{\prime}$. a priori $F^{\prime}$ is not arithmetically normal, so the singular locus of $F^{\prime}$ does not consist of isolated points and may well contain the whole curve $\Gamma$.

After the quadratic transformation (blow up) an "integral closure" transformation (normalization) is applied, so that again one deals only with finitely many isolated singular points. Applying a quadratic transformation with fundamental point at the singular point $P_{1}$ of the arithmetically normal surface $F_{1}$ that has been derived from $F^{\prime}$ followed by an "integral closure" transformation an arithmetically normal surface $F_{2}$. Continuing this procedure gives a sequence of birationally equivalent arithmetically normal surfaces $F, F_{1}, F_{2}, \ldots$ The reduction theorem as formulated by Zariski states that "After a finite number of quadratic and integral closure transformations, a normal surface $F_{i}$ is obtained which is free from singularities." [Zariski 1939b, p. 688 or 1972, p. 374]. To show that this process really ends with a surface that has no singularities, Zariski used valuation theoretic arguments. Namely, let $P, P_{1}, \ldots$ be the sequence of points obtained by the alternating transformations. The union of the quotient rings $Q(P), Q\left(P_{1}\right), \ldots$ (in modern terminology: the local rings at $\left.P_{1}, P_{2}, \ldots\right)$ is the valuation ring of a zero dimensional valuation of the function field $\Sigma$. Local uniformisation says that for every zero-dimensional valuation of $\Sigma$ there exists a projective model $F$ of $\Sigma$ on which the center of the valuation

\footnotetext{
${ }^{3}$ For a detailed historical reading of the proof as well as a deepened treatment of most of the arguments given in this chapter see [Slembek 2003].
} 
is a simple point of the surface. On the basis of this result, Zariski shows the "local reduction theorem" which states that in the sequence $P, P_{1}, P_{2}, \ldots$ a simple point occurs. As there were only finitely many points to be resolved, the process ends.

With this proof the first important step in the arithmetization process was accomplished.

To summarize, the features arithmetization process that we elucidated are the following. The programme of arithmetization aiming at the foundations of Italian algebraic geometry was undertaken by one of the schools own members. Arithmetization is a foundation of algebraic geometry that started at a precise geometric problem; it is not presented in an axiomatic way. Its main goal is not rigor because it can rely on some rigorous foundations. Its goal is a conservative one.

But even if the aim of arithmetization was to legitimize and to preserve the Italian tradition of algebraic geometry, this was achieved only by completely reshaping algebraic geometry and finally by replacing its methods and thus changing it into a different discipline.

\section{References}

Baker, Henry Frederick. 1913. "On some recent advances in the theory of algebraic surfaces." Proceedings of the London Mathematical Society, Ser. 2, Vol. 12, 1-40.

Brill, Alexander von and Noether, Max. 1874. "Ueber die algebraischen Functionen und ihre Anwendung in der Geometrie." Mathematische Annalen 7, 269-310.

Brigaglia, Aldo and Ciliberto, Ciro. 1995. Italian algebraic geometry between the two world wars. Trans. Jeanne Duflot. Queen's Papers in Pure and Applied Mathematics 100. Queen's University, Kingston, ON.

Castelnuovo, Guido and Enriques, Federigo. 1897. "Sur quelques récents résultats dans la théorie des surfaces algébriques." Mathematische Annalen 48, 241-316.

Corry, Leo. 1996. Modern algebra and the rise of mathematical structures. Basel: Birkhäuser.

Gario, Paola. 1988. "Histoire de la résolution des singularités des surfaces algébriques (une discussion entre C. Segre et P. Del Pezzo)." Cahiers du Séminaire d'Histoire des Mathématiques 9, 123-137.

Gario, Paola. 1989. "Resolution of singularities of surfaces by P. Del Pezzo. A mathematical controversy with C. Segre." Archive for the History of Exact Sciences 40. no. 3, 247-274.

Mumford, David. 1986. "Oscar Zariski 1899-1986." Notices of the American Mathematical Society 33, 891-894.

Parikh, Carol. 1991. The unreal life of Oscar Zariski. San Diego: Academic Press. 
Segre, Corrado. 1894. "Introduzione alla geometria sopra un ento algebrico semplicemente infinito." Annali di matematica pura ed applicata, Ser. 2, Vol. 22, 41-142 or Opere. 4 Vols. 1957-1963. Rome: Edizione Cremonese. Vol. 1, 198-304.

Severi, Francesco. 1949. "La géométrie algébrique italienne. Sa rigueur, ses méthodes, ses problèmes." Coll. de Géom. algébrique, Liège

Slembek, Silke. 2003. "Continuité ou rupture? A propos de l'arithmétisation de la géométrie algébrique selon Oscar Zariski." Ph. D. Thesis. Preprint. http://www-irma.u-strasbg.fr/irma/publications/2003/03005.shtml

Veronese, Giuseppe. 1882. "Behandlung der projectivischen Verhältnisse der Räume von verschiedenen Dimensionen durch das Princip des Projicirens und Schneidens." Mathematische Annalen 19, 161-234.

Walker, Robert J. 1935. "The reduction of the singularities of an algebraic surface." Annals of Mathematics 36, 336-365.

Zariski, Oscar. 1935. Algebraic Surfaces. Berlin: Springer.

Zariski, Oscar. 1938. "Polynomial ideals defined by infinitely near base points." American Journal of Mathematics, 60, 151-204, or Oscar Zariski: Collected Papers. 4 Vols. Cambridge: MIT Press, 1972-1979. Vol. 1, Ed. Heisuke Hironaka and David Mumford, 14-67.

Zariski, Oscar. 1939a. "Some results in the arithmetic theory of algebraic varieties." American Journal of Mathematics 61, 249-294 or Oscar Zariski: Collected Papers. 4 Vols. Cambridge: MIT Press, 1972-1979. Vol. 1, Ed. Heisuke Hironaka and David Mumford, 73-118.

Zariski, Oscar. 1939b. "The reduction of the singularities of an algebraic surface." Annals of Mathematics 40 or Oscar Zariski: Collected Papers. 4 Vols. Cambridge: MIT Press, 1972-1979. Vol. 1, Ed. Heisuke Hironaka and David Mumford, 639-689.

Zariski, Oscar. 1950. "The fundamental ideas of abstract algebraic geometry." Proceedings of the ICM, Cambridge, Mass., 77-89. or Oscar Zariski: Collected Papers. 4 Vols. Cambridge: MIT Press, 1972-1979. Vol. 3, Ed. Michael Artin and Barry Mazur, 363-375. 